General Relativity and Gravitation, Vol. 31, No. 4, pp. 571-577, 1999

\title{
LETTER
}

\section{Classical and Quantum Evolution of Non-Isentropic Hot Singular Layers in Finite-Temperature General Relativity}

\author{
Konstantin G. Zloshchastiev"
}

Received December 14, 1998

\begin{abstract}
The spherically symmetric layer of matter is considered within the frameworks of general relativity. We perform generalization of the already known theory for the case of nonconstant surface entropy and finite temperature. We also propose the minisuperspace model to determine the behavior of temperature field and perform the Wheeler-DeWitt quantization.

PACS number(s): 04.20.Fy, 04.40.Nr, 04.60.Kz, 11.27.+d
\end{abstract}

KEY WORDS : Singular layer ; general relativity ; minisuperspace quantization

Beginning with the outstanding classic works (see Ref. [1] and references therein) the theory of singular hypersurfaces has been intensively developed both in the axiomatic and applied aspects [2]. Regrettably, the majority of recent investigations (especially quantum) mainly touch upon the subject of the temperatureless theory of isentropic thin shells. However, considering thermal effects should always increase the physical relevance of any approach and expand the domain of its applicability. Therefore, the aim of present letter is to study the non-isentropic singular layers at finite temperature within the frameworks of classical and quantum general relativity.

\footnotetext{
${ }^{1}$ Postal address: Box 2837, Dnepropetrovsk 320128, Ukraine. E-mail: zlosh@email.com. URL(s): http://zloshchastiev.webjump.com, http://zloshchastiev.cjb.net
} 
So, one considers an infinitely thin layer of matter with the thermally generalized surface stress-energy tensor of a perfect fluid

$$
\mathcal{S}_{a b}=\left(\sigma-\frac{1}{A} \int T \mathrm{~d} S\right) u_{a} u_{b}+p\left(u_{a} u_{b}+{ }^{(3)} g_{a b}\right),
$$

where $\sigma$ and $p$ are respectively the surface energy density and pressure, $u^{a}$ is the timelike unit tangent vector, ${ }^{(3)} g_{a b}$ is the three-dimensional world sheet metric on the layer; $A, S$ and $T$ are surface area, entropy and temperature respectively.

Assuming the layer $\Sigma$ to be spherical we suppose the metrics of spacetimes outside $\Sigma^{+}$ and inside $\Sigma^{-}$in the special form

$$
\mathrm{d} s_{ \pm}^{2}=-\Phi^{ \pm}(r) \mathrm{d} t_{ \pm}^{2}+\Phi^{ \pm}(r)^{-1} \mathrm{~d} r^{2}+r^{2} \mathrm{~d} \Omega^{2}
$$

where $d \Omega^{2}=\mathrm{d} \theta^{2}+\sin ^{2} \theta \mathrm{d} \varphi^{2}$. Of course, with this we have some loss of generality but the assumption (2) turns to be enough for major physically interesting tasks. In terms of the layer's proper time $\tau$ the world sheet three-metric is

$$
{ }^{(3)} \mathrm{d} s^{2}=-\mathrm{d} \tau^{2}+R^{2} \mathrm{~d} \Omega^{2},
$$

where $R=R(\tau)$ is a proper radius of the layer. The Einstein equations can be decomposed as the jump of extrinsic curvatures across the singular layer

$$
\left(K_{b}^{a}\right)^{+}-\left(K_{b}^{a}\right)^{-}=4 \pi \sigma\left(2 u^{a} u_{b}+\delta_{b}^{a}\right) .
$$

For the spacetimes (2) after straightforward computing the $\theta \theta$ component of this equation yields the equation of layer's radial motion

$$
\epsilon_{+} \sqrt{\dot{R}^{2}+\Phi^{+}}-\epsilon_{-} \sqrt{\dot{R}^{2}+\Phi^{-}}=-m / R
$$

where $\Phi^{ \pm}=\left.\Phi^{ \pm}(r)\right|_{r=R(\tau)}, \dot{R}=\mathrm{d} R / \mathrm{d} \tau$ is the radial velocity, $\epsilon_{ \pm}=\operatorname{sgn}\left[\sqrt{1+\dot{R}^{2}+\Phi^{ \pm}}\right]$, $m=4 \pi \sigma R^{2}$ is interpreted as the (effective) rest mass. The sign $\epsilon=+1$ if $R$ increases in the outward normal of a layer, and $\epsilon=-1$ if $R$ decreases. Besides, double squaring we can write eq. (5) in the form

$$
\dot{R}^{2}=\left[\frac{\Delta \Phi-m^{2} / R^{2}}{2 m / R}\right]^{2}-\Phi^{-}
$$

where $\Delta \Phi=\Phi^{+}-\Phi^{-}$.

The necessary condition of integrability of the Einstein equations is the energy conservation law $\mathcal{S}_{b ; a}^{a}=0$ for matter on the layer. Using eqs. (1), (3) and the property

$$
\frac{4 \pi}{\sin \theta} \sqrt{\left|\operatorname{det}\left({ }^{(3)} g_{a b}\right)\right|}=4 \pi R^{2}=A,
$$

it can be represented as the first thermodynamical law

$$
\mathrm{d}(\sigma A)+p \mathrm{~d} A-T \mathrm{~d} S+A \Delta T^{\tau n} \mathrm{~d} \tau=0,
$$

where $\Delta T^{\tau n}=\left(T^{\tau n}\right)^{+}-\left(T^{\tau n}\right)^{-}, T^{\tau n}=T^{\alpha \beta} u_{\alpha} n_{\beta}$ is the projection of the stress-energy tensors in the $\Sigma^{ \pm}$spacetimes on the tangent and normal vectors. It can be checked immediately that for spacetimes (2) $T^{\tau n} \equiv 0$, and we have a conservative system

$$
\mathrm{d}(\sigma A)+p \mathrm{~d} A-T \mathrm{~d} S=0 .
$$


We will assume the layer's temperature as the internal degree of freedom which appears to be complementary to radius (or, equivalently, to area). Following the definition of the entropy as a state function of temperature and area, we obtain

$$
\mathrm{d} S=\frac{\partial S}{\partial T} \mathrm{~d} T+\frac{\partial S}{\partial A} \mathrm{~d} A
$$

Comparing eqs. (9) and (10), we obtain

$$
\begin{aligned}
& \frac{\partial S}{\partial T}=\frac{A}{T} \frac{\partial \sigma}{\partial T}, \\
& \frac{\partial S}{\partial A}=\frac{1}{T}\left[p+\frac{\partial(\sigma A)}{\partial A}\right] .
\end{aligned}
$$

Then the equality of mixed derivatives yields the partial differential equation

$$
\frac{\partial \sigma}{\partial A}-T \frac{\partial p}{\partial T}+p+\sigma=0
$$

which is useful both for obtaining the internal energy as a function of area and temperature from a known equation of state and for the inverse problem. Thus, eqs. (5), (9) and (13) together with an equation of state and choice of signs $\epsilon^{ \pm}$are almost sufficient for finding of all the unknowns. The equation completing this system is that for temperature field, and seems to be introduced from some additional assumptions. One of them is the variational minisuperspace model which will be proposed below.

Let us choose now an equation of state of layer's 2D matter. The simplest (but physically most interesting) EOS is the linear one of barotropic fluid,

$$
p=\eta \sigma,
$$

then the quasilinear PDE (13) has the general solution

$$
\sigma=f\left(A^{\eta} T\right) A^{-\eta-1}
$$

where $f$ is an arbitrary function which can be determined by means of initial conditions, correspondence principle, etc. For instance, at $\eta \neq 0$ one can assume

$$
f^{(p h)}(x)=\alpha+\beta x^{1+1 / \eta},
$$

where $\alpha$ and $\beta$ are some constants. Therefore,

$$
\sigma^{(p h)}=\frac{\alpha}{A^{\eta+1}}+\beta T^{1+1 / \eta}
$$

and this expression will reproduce as limiting cases both the already known theory of isentropic thin shells at $\beta=0$ and the thermodynamics of a homogeneous 2D fluid $\sigma=\sigma(T)$ at $\alpha=0$. Keeping in mind $\sigma^{(p h)}$, we nevertheless will attempt to construct the theory for arbitrary $f\left(A^{\eta} T\right)$.

First of all, from eq. (9) one can obtain the relation between entropy (which has to be a function of $A^{\eta} T$ as well) and $f$ :

$$
A^{\eta} T \frac{\mathrm{d} S}{\mathrm{~d} f}=1
$$


which (i) says that surface entropy can be found (up to additive constant) if we know $f$ explicitly, (ii) imposes, through the second law of thermodynamics, the restriction of positive monotonicity of $f: \mathrm{d} f \geq 0$. For example, following (i) for $f=f^{(p h)}$ we obtain

$$
S^{(p h)}=\beta(\eta+1) A T^{1 / \eta}+S_{0},
$$

where (ii) $\beta$ is restricted:

$$
\beta\left\{\begin{array}{l}
\geq 0 \text { at } \eta \in(-\infty,-1) \cup[0,+\infty), \\
\leq 0 \text { at } \eta \in(-1,0), \\
\text { arbitrary at } \eta=-1 .
\end{array}\right.
$$

Let us recall now the above-mentioned problem of the missing equation for the temperature field. We will suppose the set of all the world sheet metrics (3) and accompanying fields to be a minisuperspace in the sense of the Wheeler-DeWitt one. It is evident that spherically symmetric world sheet metrics is determined by a single function $R(\tau)$, and hence we can consider on this minisuperspace the model described by the following action

$$
\mathcal{A}=\int \mathcal{L} \mathrm{d} \tau, \quad \mathcal{L}=L w,
$$

where $w=w(R, T)$ is an arbitrary gauge function, and

$$
L=\frac{m \dot{R}^{2}}{2}-\frac{m}{2}\left[\Phi^{-}-\left(\frac{\Delta \Phi-m^{2} / R^{2}}{2 m / R}\right)^{2}\right],
$$

in which temperature and radius are considered as independent generalized coordinates. Note that the gauge $w=1$ seems to be the most physically justified but other gauges (e.g., $w=2 m_{\text {Planck }} / m$ ) are not evidently forbidden, and therefore we will work with arbitrary $w$.

Extremalizing the action with respect to radius, $\delta \mathcal{A} / \delta R=0$, we obtain the equation of radial motion

$$
\frac{\mathrm{d}(m w \dot{R})}{\mathrm{d} \tau}=\frac{(m w)_{, R} \dot{R}^{2}}{2}-w\left[\frac{m \Phi^{-}}{2}-\left(\frac{\Delta \Phi-m^{2} / R^{2}}{2 \sqrt{2 m} / R}\right)^{2}\right]_{, R},
$$

where subscript ", $x$ " means the partial derivative with respect to $x$. Using time symmetry, we can decrease an order of this ordinary differential equation to obtain

$$
\dot{R}^{2}=\frac{2 H}{m w}-\Phi^{-}+\left[\frac{\Delta \Phi-m^{2} / R^{2}}{2 m / R}\right]^{2}
$$

where $H$ is an integration constant. Supposing it to be vanishing on real trajectories (thus one has the constraint $H \approx 0$ ) we obtain the equation of motion (6). Thus, our Lagrangian indeed describes dynamics of the layer up to topological features which were described by the signs $\epsilon_{ \pm}$. However, we can always restore the topology $\epsilon_{ \pm}$both at classical levels (rejecting redundant roots) and at quantum levels (considering appropriate boundary conditions for the corresponding Wheeler-DeWitt equation).

Further, extremalizing the action with respect to temperature, $\delta \mathcal{A} / \delta T=0$, and taking into account the equation of radial motion, we obtain the equation for the temperature field $T(R)$ :

$$
\left(\Phi^{+}+\Phi^{-}-\phi\right) \phi_{, T}-\left[\Delta \Phi^{2}-2 \phi\left(\Phi^{+}+\Phi^{-}\right)+\phi^{2}\right](\ln w)_{, T}=0
$$


where $\phi=(4 \pi \sigma R)^{2}=4 \pi A^{-2 \eta-1} f^{2}\left(A^{\eta} T\right)$. It is clear that this expression is nothing but the constraint for the temperature as a non-independent degree of freedom. Therefore, for every physical concrete case one should resolve eq. (25) with respect to $T$ as a function of radius and substitute it into the initial Lagrangian. Thus, the radius remains the only canonical variable. Further, one can see that gauge function $w$ does not affect the radial motion but appears in the temperature field equation. (Moreover, it will be shown below that $w$ affects also on quantum dynamics.) Thus, the problem of obtaining a missing temperature equation has been reduced to that of $w$ choice within the frameworks of the minisuperspace model hypothesis.

Now we have all required expressions to perform the Wheeler-DeWitt quantization of our model [3]. Of course, such a quantization is not the only way (see Ref. [4], and references therein). However, in the absence of a rigorous axiomatic approach this method has many advantages in comparison with others [5], as follows. (i) Quantum dynamics can be constructed independently of time slicing on the basic patchwork manifold $\Sigma^{+} \cup \Sigma \cup \Sigma^{-}$ (strictly speaking, such a union space does not have be the manifold in the conventional sense). (ii) This method is simple and gives many heuristic results in a nonperturbative way, which is very important for non-linear general relativity. (iii) There explicitly exists conformity with the correspondence principle that improves physical interpretation of all the concepts of the theory.

According to eqs. (21), (22) the canonical momentum conjugated to radius is

$$
\Pi_{R}=m w \dot{R}
$$

and hence the super-Hamiltonian has to be

$$
\mathcal{H}=\Pi_{R} \dot{R}-\mathcal{L} \equiv w H=0,
$$

where the temperature-field constraint should be already taken into account. In the quantum case $\hat{\Pi}_{R}=-i \partial / \partial R$ (we assume Planckian units) it yields the Wheeler-DeWitt equation for a wave function $\Psi(R, T(R))$ describing quantum oscillations of the layer surface,

$$
\frac{\partial^{2} \Psi}{\partial R^{2}}+m^{2} w^{2}\left[\left(\frac{\Delta \Phi-m^{2} / R^{2}}{2 m / R}\right)^{2}-\Phi^{-}\right] \Psi=0,
$$

from which one can obtain eigenfunctions and spectra for all concrete physical values which can appear in the theory.

Let us summarize briefly the main points studied. We generalized the theory of singular hypersurfaces for the case of the nonconstant surface entropy and finite temperature. Then we introduced the minisuperspace model which, firstly, provided a variational procedure for describing the temperature field, and, secondly, determined all the canonical variables necessary for the Wheeler-DeWitt's quantization of the theory. 


\section{References}

[1] Israel, W. (1966). Nuovo Cimento B44, 1.

[2] Misner, C. W., Thorne, K. S., and Wheeler, J. A. (1973). Gravitation (W. H. Freeman, San Francisco).

[3] Vilenkin, A. (1994). Phys. Rev. D50, 2581.

[4] Nakamura, K., Oshiro, Y., and Tomimatsu, A. (1996). Phys. Rev. D54, 4356.

[5] Hájíček, P., Kay, B. S., and Kuchař, K. V. (1992). Phys. Rev. D46, 5439;

Zloshchastiev, K. G. (1998). Phys. Rev. D57, 4812."

${ }^{2}$ See also Zloshchastiev, K. G. (1999). Int. J. Mod. Phys. D8, 165;

- (1999). Int. J. Mod. Phys. D8, 363;

- (1999). Class. Quantum Grav. 16, 1737;

- (1997). LANL e-print gr-qc/9709030. 